Research Article

\title{
Multidimensional System Identification and Active Vibration Control of a Piezoelectric-Based Sting System Used in Wind Tunnel
}

\author{
Yi Yu $\mathbb{D}^{\mathbb{D}}$, Xing Shen $\mathbb{D}$, and Yun Huang \\ State Key Laboratory of Mechanics and Control of Mechanical Structures, Nanjing University of Aeronautics and Astronautics, \\ Nanjing 210016, China \\ Correspondence should be addressed to Xing Shen; shenx@nuaa.edu.cn
}

Received 1 July 2020; Revised 3 September 2020; Accepted 7 September 2020; Published 19 September 2020

Academic Editor: Abdullah Seçgin

Copyright (c) 2020 Yi Yu et al. This is an open access article distributed under the Creative Commons Attribution License, which permits unrestricted use, distribution, and reproduction in any medium, provided the original work is properly cited.

In wind tunnel tests, the cantilever sting is usually used to support aircraft models because of its simple structure and low aerodynamic interference. However, in some special conditions, big-amplitude and low-frequency vibration would occur easily on the model not only in the pitch direction but also in the yaw direction, resulting in inaccurate data and even damage of the supporting structure. In this paper, aiming at suppressing the vibration in pitch and yaw plane, a multidimensional system identification and active vibration control system on the basis of piezoelectric actuators is established. A vibration monitoring method based on the strain-displacement transformation (SDT) matrix is proposed, which can transform strain signals into vibration displacements. The system identification based on chirp-Z transform (CZT) is applied to improve the adaptability and precision of the building process for the system model. After that, the hardware platform as well as the software control system based on the classical proportional-derivative (PD) algorithm is built. A series of experiments are carried out, and the results show the exactness of the vibration monitoring method. The system identification process is completed, and the controller is designed. Vibration control experiments verify the effectiveness of the controller, and the results indicate that vibrations in pitch and yaw directions are attenuated apparently. The spectrum power is reduced over $14.8 \mathrm{~dB} / \mathrm{Hz}$, which prove that the multidimensional identification and active vibration control system has the capability to decline vibration from different directions.

\section{Introduction}

Wind tunnel tests, through which precise aerodynamic characteristics and accurate experimental data of the model can be obtained, play an important role in designing the structure of spacecraft or aircraft [1]. In order to reduce the aerodynamic interference around wind tunnel models, a long cantilever sting is widely used to support the model. However, the cantilever sting is intended from 3 to 5 times longer than the test model, which suggests its inherent low structural damping [2]. When the aircraft model sweeps to large angle-of-attack, vibration would occur easily on the model both in pitch and yaw directions, which is harmful for the experimental data [3]. Therefore, aiming at suppressing the vibration, many scholars have tried different control methods.
Generally, there are two kinds of vibration control methods, namely, passive methods and active methods. In 1968, Igoe and Capone [4] first proposed the passive control method using a tuned damped vibration absorber installed on the model. Then, multiple tuned mass dampers [5] and viscoelastic shear lap dampers [6] were also adopted in engineering. However, these absorbers need to be tuned specifically, which means they have low compatibility and cannot adapt to all experimental conditions. With the swift development of smart materials and structures, more and more active vibration methods have been discussed all over the world. In autumn 1994, Fehren [7] performed the first successful experiment using an active antivibration system (AVS) being excited by piezoceramic elements. Based on this system, a series of tests were carried out in Europe transonic wind tunnel (ETW) $[8,9]$. The results show that although the 
structure can work at high pressure and cryogenic temperatures, its vibration control effect is only approximately $60 \%$ and the ability to suppress high-amplitude vibrations is poor. Meanwhile, many research studies on active vibration control have been investigated at the NASA Langley Research Center National Transonic Facility (NTF). In 2007, Balakrishna et al. [10] designed a sting damper by use of piezoceramic actuator cluster and wind tunnel tests showing that large vibrations can be reduced typically and attenuation of mode response amplitudes has also been realized. Depending on the closed loop control system, an average peak attenuation of about $10 \mathrm{~dB}$ and vibration attenuation of about $5 \mathrm{~dB}$ were gained by changing the piezocluster configuration [11]. Acheson and Balakrishna [12] conducted many active control experiments on the common research model (CRM) at the NTF, and large sets of data showed that active damping does not negatively affect aerodynamic coefficient data, which makes a good case for widespread implementation of active damping in transonic testing. Moreover, test data from CRM wind tunnel evaluation [13] showed that the NTF designed piezoactive damper has overcome sting divergent oscillations and has permitted a bigger angle-of-attack at low temperatures. However, although the vibration of the model can be attenuated, the performance and functionality of active controllers depend on control algorithms [14]. Shen et al. [2, 15-18] used the classical PD algorithm, linear quadratic regulator (LQR) optimal control algorithm, and other algorithms aiming at controlling the vibration of the wind tunnel aircraft model in the pitch direction. The experiments suppressed over $90 \%$ of the vibration amplitude, and the power spectrum density (PSD) was reduced at least $26 \mathrm{~dB} / \mathrm{Hz}$. Liu et al. [3, 19] applied a root mean square (RMS) evaluation method, and the results indicate that the damping ratio is improved more than 4 times.

Up to now, the research on vibration control has made great progress. However, there are still some problems that need to be solved. Currently, there are two main sensing processes used to measure the vibration signals, namely, the use of accelerometers and test balances. Accelerometers need to be embedded in the model or attached to the surface of the model, which will destroy the structure of the model or the aerodynamic layout of the surface, sometimes resulting in inaccurate experimental data. The test balance is mainly composed of elastic element, resistance strain gauge, and measuring circuit. In the wind tunnel experiment, the balance bears the aerodynamic load acting on the model and transmits it to the support system. However, the balance is generally installed at the tip of the sting, which means it cannot measure the maximum strain. The traditional calculation of motion equations of the system has also certain disadvantages. Many unexpected factors like the level of noise or subtle misalignment during assembly can have a great impact on the establishment of system models and transfer functions. Sometimes, changing a model may result in different terms in the equation, which brings difficulties and inconvenience to the setting of control parameters. Last but not least, most studies have only suppressed the vibration in the pitch direction. Due to lack of a precise vibration monitoring method and suitable vibration control system, multidimensional vibration control is explored little. However, for circular symmetric wind tunnel models such as missiles, the vibration is not only just in the pitch direction but also in the yaw direction under certain circumstances, which is also one aspect that must be taken into account. In this paper, to suppress the vibration from pitch and yaw directions, a multidimensional system identification and active vibration control system on the basis of piezoelectric actuators is established.

The remainder of this article is organized as follows. In Section 2, a vibration monitoring method based on SDT matrix is proposed, through which the position of the sensor can be optimized to accurately measure the displacement of vibration in pitch and yaw directions. Section 3 presents the multidimensional system identification based on CZT to improve the adaptability and precision of the system model building process. In Section 4, the hardware platform and the real-time software control system of the wind tunnel sting model is built. The exactness of the vibration monitoring method and system identification process is proved, and the first-order vibration in pitch and yaw directions is finally attenuated apparently. Section 5 summarizes the paper.

\section{Modeling of the Sting and the Vibration Monitoring Method}

The support structure used in wind tunnel experiments is generally composed of a long cantilever sting, a test balance, several piezoelectric stacks, and bolts for connection (Figure 1). The aircraft model is assembled at the tip of the sting, which is exposed to wide frequency aerodynamic load during the tests. The load arouses dynamic bending in both pitch and yaw planes, while restoring moment should be generated by the piezoelectric stack installed at the bottom of the sting to offset the effect of loading moment. The system can be considered as a mass-spring system, and the vibration equation can be expressed as

$$
\mathrm{M} \ddot{X}+\dot{\mathrm{CX}}+\mathrm{KX}=F
$$

where $M, C, K$, and $F$ are the modal mass matrix, damping matrix, stiffness matrix, and force vector of the aerodynamic load. These matrices are expressed as

$$
\begin{aligned}
X & =\left[\begin{array}{l}
d_{y} \\
d_{z}
\end{array}\right], \\
M & =\left[\begin{array}{cc}
M_{y} & 0 \\
0 & M_{z}
\end{array}\right], \\
K & =\left[\begin{array}{cc}
K_{y} & 0 \\
0 & K_{z}
\end{array}\right], \\
C & =\left[\begin{array}{cc}
C_{y} & 0 \\
0 & C_{z}
\end{array}\right],
\end{aligned}
$$

where $M_{y}, C_{y}, K_{y}, M_{z}, C_{z}$, and $K_{z}$ are the modal mass matrices, damping matrices, and stiffness matrices of the 
system, respectively, in pitch plane and yaw plane; $d_{y}$ and $d_{z}$ are the pitch displacement and yaw displacement, respectively.

It is obvious that $d_{y}$ and $d_{z}$ are indispensable parameters to obtain the vibration equation. In order to solve the problem mentioned above, we need to find the relationship between strain and displacement and optimize the location of strain gauges. A vibration monitoring method based on the SDT matrix is proposed here, which will not affect the model, while the vibration displacement can be measured without complex external instruments.

Every vibrating structure in the modal space can be divided into infinite number of mode shapes, and each mode has different weighting factors [20]. These weighting factors are called modal coordinates $\eta$. For the sake of convenience, we first only consider the displacement, strain, and modes in the pitch direction. So, the displacement $d$ can be expressed by $n$ mode shapes:

$$
\{d\}_{N \times 1}=[\phi]_{N \times n} \cdot\{\eta\}_{n \times 1} .
$$

Therefore, $[\phi]_{N \times n}$ is the displacement modal matrix and $N$ is the number of points whose displacement is concerned. And the strain $\varepsilon$ can also be expressed as

$$
\{\varepsilon\}_{M \times 1}=[\phi]_{M \times n} \cdot\{\eta\}_{n \times 1},
$$

where $[\phi]_{M \times n}$ is the strain modal matrix and $M$ is the number of points whose strain is concerned. It can be seen that the same modal coordinates are used for the strain mode shapes as well as for the deformation mode shapes. Using some transformations, we get

$$
\begin{gathered}
\{\eta\}_{n \times 1}=\left([\phi]_{N \times n}^{T}[\phi]_{N \times n}\right)^{-1}[\phi]_{N \times n}^{T} \cdot\{d\}_{N \times 1}, \\
\{\eta\}_{n \times 1}=\left([\phi]_{M \times n}^{T}[\phi]_{M \times n}\right)^{-1}[\phi]_{M \times n}^{T} \cdot\{\varepsilon\}_{M \times 1} .
\end{gathered}
$$

Substituting equation (5) into equation (4) and equation (6) into equation (3), we get

$$
\begin{aligned}
\{d\}_{N \times 1} & =[\phi]_{N \times n}\left([\phi]_{M \times n}^{T}[\phi]_{M \times n}\right)^{-1}[\phi]_{M \times n}^{T} \cdot\{\varepsilon\}_{M \times 1}, \\
\{\varepsilon\}_{M \times 1} & =[\phi]_{M \times n}\left([\phi]_{N \times n}^{T}[\phi]_{N \times n}\right)^{-1}[\phi]_{N \times n}^{T} \cdot\{d\}_{N \times 1} .
\end{aligned}
$$

Let

$$
\begin{aligned}
{[\mathrm{SDT}]_{N \times M} } & =[\phi]_{N \times n}\left([\phi]_{M \times n}^{T}[\phi]_{M \times n}\right)^{-1}[\phi]_{M \times n}^{T}, \\
{[\mathrm{DST}]_{M \times N} } & =[\phi]_{M \times n}\left([\phi]_{N \times n}^{T}[\phi]_{N \times n}\right)^{-1}[\phi]_{N \times n}^{T} .
\end{aligned}
$$

Then, we obtain

$$
\begin{aligned}
& \{d\}_{N \times 1}=[\mathrm{SDT}]_{N \times M} \cdot\{\varepsilon\}_{M \times 1}, \\
& \{\varepsilon\}_{M \times 1}=[\mathrm{DST}]_{M \times N} \cdot\{d\}_{N \times 1},
\end{aligned}
$$

where $[\mathrm{SDT}]_{N \times M}$ means strain to displacement transformation matrix and [DST $]_{M \times N}$ means displacement to strain transformation matrix. Shown in Figure 1(a), the displacement of which we concerned is the center of gravity $d_{g}$ and tip of the sting $d_{t}$. Because the energy of vibration is mainly concentrated in the first few modes, we only consider the first two modes in each direction, so equation (9) can be written as

$$
\left\{\begin{array}{l}
d_{g} \\
d_{t}
\end{array}\right\}=[\mathrm{SDT}] \cdot\left\{\begin{array}{l}
\varepsilon_{1} \\
\varepsilon_{2}
\end{array}\right\},
$$

where $\varepsilon_{1}$ and $\varepsilon_{2}$ are any two points where strain can be obtained on the cantilever sting and [SDT] here is a $2 * 2$ matrix related to the selection of $\varepsilon_{1}$ and $\varepsilon_{2}$.

The condition number of a matrix $\mathrm{A}$ is defined as

$$
\mathrm{CN}(A)=\|A\| \cdot\left\|A^{-1}\right\| \text {, }
$$

where $A$ is the matrix norm of $\mathrm{A}$. The condition number $\mathrm{CN}$ is an index for the information conservation during matrix operations. The minimum possible $\mathrm{CN}$ is 1 , which indicates the best information keeping. The larger the condition number $\mathrm{CN}$, the worse the information conservation. In this paper, the condition number $\mathrm{CN}$ of the SDT matrix is related to the position of the strain gauge, which needs to be optimized in order to improve the estimated results of vibration displacement. To get the displacement modal matrix and strain modal matrix, the finite element simulation method is used here. Using ANSYS to carry out structural modal analysis of the model support system, the first four order vibration modes are shown in Figure 2. The modal frequencies in the yaw direction are $17.67 \mathrm{~Hz}$ and $123.27 \mathrm{~Hz}$, respectively, and in the pitch direction are $18.31 \mathrm{~Hz}$ and $272.62 \mathrm{~Hz}$.

And the displacement modal matrix and strain modal matrix can be exported. Improved particle swarm optimization (PSO) algorithm [21] is used to launch optimization calculation. The improved velocity update formula can be expressed by

$$
\begin{aligned}
V_{\mathrm{id}}(k+1)= & \omega \cdot V_{\mathrm{id}}(k)+c_{1} r_{1}\left[P_{\mathrm{id}}-x_{\mathrm{id}}(k)\right] \\
& +c_{2} r_{2}\left[P_{\mathrm{gd}}-x_{\mathrm{id}}(k)\right] \\
\omega= & \left(w_{\max }-\omega_{\min }\right) \cdot \frac{T_{\max }-N}{T_{\max }}+\omega_{\min },
\end{aligned}
$$

where $V_{\text {id }}(k)$ is the $d$-th component of the velocity vector at the $k$-th iteration of the particle $i ; x_{\text {id }}(k)$ is the $d$-th component of the position vector at the $k$-th iteration of the particle $i(i=1,2, \ldots, n, d=1,2, \ldots, D) ; k$ is the number of iterations; $c_{1}$ and $c_{2}$ are the learning factors; $r_{1}$ and $r_{2}$ are uniformly distributed random numbers in the range $[0,1]$; $P_{\text {id }}$ is the $d$ th component of the historical optimal position of particle $i ; P_{\mathrm{gd}}$ is the $d$ th component of the global optimal position; $\omega$ is the inertial weight variable; $\omega_{\min }$ and $\omega_{\max }$ are the minimum and maximum of inertia weight $\left(0.1 \leq \omega_{\min }<\omega_{\max } \ll 0.9\right)$; and $T_{\max }$ and $N$ are the maximum iteration number and current iteration number, respectively.

Then, strain sensor positions were optimized in the range of 0 to $350 \mathrm{~mm}$ by the improved PSO algorithm. The number of particles $n$ was set 20 , the dimension $D$ was set 2 , both of the learning factors $c_{1}$ and $c_{2}$ were set 2 , and the maximum iteration number $T_{\max }$ was set 50 . In addition, the minimum 


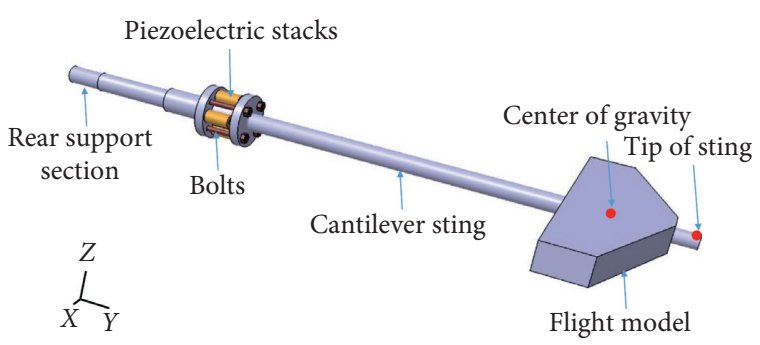

(a)

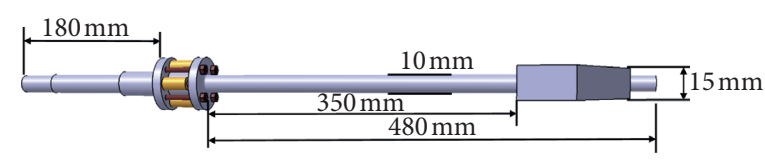

(b)

Figure 1: Schematic diagram of the sting: (a) diagram of the sting; (b) main dimensions of the sting.

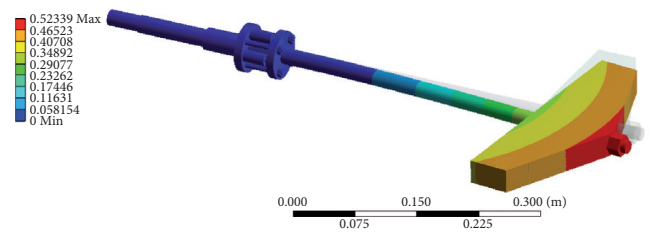

(a)

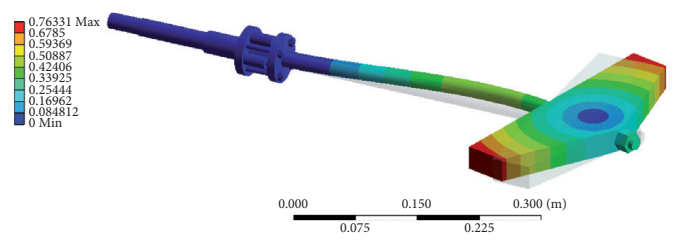

(c)

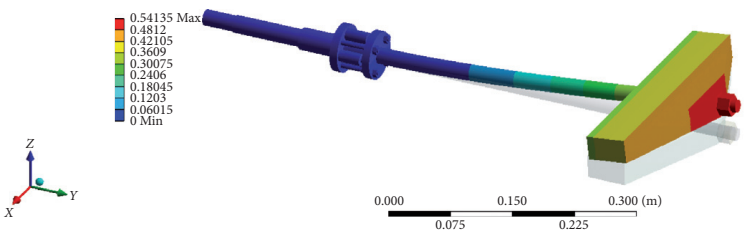

(b)
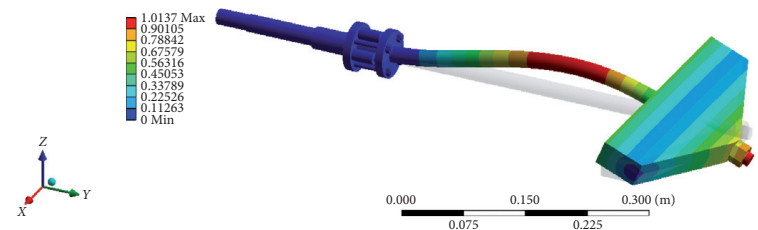

(d)
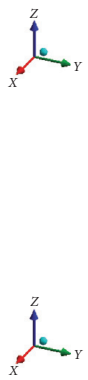

FIgUre 2: The first four modes of the system: (a) 1st mode shape $(17.67 \mathrm{~Hz})$; (b) 2nd mode shape $(18.31 \mathrm{~Hz})$; (c) 3rd mode shape (123.27 Hz); (d) 4 th mode shape $(272.62 \mathrm{~Hz})$.

and maximum of inertia weights $\omega_{\min }$ and $\omega_{\max }$ were set 0.4 and 0.9 , respectively. Furthermore, boundary conditions that limit the velocity and position of particles were also set to guarantee the convergence of the algorithm. The velocity range was $[-1,1]$, and the position range was $[0,350]$. The fitness iteration curve of the optimal particle is shown in Figure 3.

The final strain sensor positions and the SDT matrices are shown in Table 1. Figure 4 shows positions of strain gauges, and the verification test is given in Section 4 .

\section{Multidimensional System Identification for Vibration Control System}

In order to design an effective controller, the vibration modes and frequency response function (FRF) of the sting structure should be determined by system identification. A certain FRF can be calculated by the following equation:

$$
H(\omega)=\frac{G_{\mathrm{xy}}(\omega)}{G_{\mathrm{xx}}(\omega)},
$$

where $G_{\mathrm{xx}}(\omega)$ is the autopower spectrum for the input signal $x(s)$ and $G_{\mathrm{xy}}(\omega)$ is the cross-power spectrum for $x(s)$ and the output signal $y(\mathrm{~s})$. It is obvious that the result $H(\omega)$ contains both amplitude and phase information. Thus, the parameters of PD controller can be found once the amplitude and phase at the mode frequencies are obtained.
Figure 5 shows several steps of designing a multidimensional vibration control system.

First the modal identification is completed by broadband white noise excitation which is generated by the piezoelectric stacks. Through analyzing the power spectral density (PSD) map of the signals from strain gauges, the first modes of pitch and yaw directions can be gained. Then, to get more accurate signals, narrow-band excitation around the modal frequency is implemented. Using the input signal and the excitation signal, FRF of the system can be established by different algorithms.

Discrete Fourier transform (DFT) is one of the most important cornerstones of digital signal processing and one of the most commonly used tools when analyzing and processing signals. However, DFT of a finite-length sequence is actually $N$ Z-transformed sampling points evenly distributed within $2 \pi$ of the unit circle, resulting in limited resolution. The method of spectrum refinement analysis is generally implemented in two ways: zoom-FFT algorithm and CZT-based spectrum thinning algorithm. CZT is suitable for narrow-band high-resolution calculations and is a classic frequency domain refinement method. Zoom-FFT is easily affected by filter performance and is difficult to adjust frequency, while the CZT-based refinement method has a simple processing process, flexible selection of refinement multiples, and high computational efficiency, which can achieve a better refinement effect for the entire frequency band. 


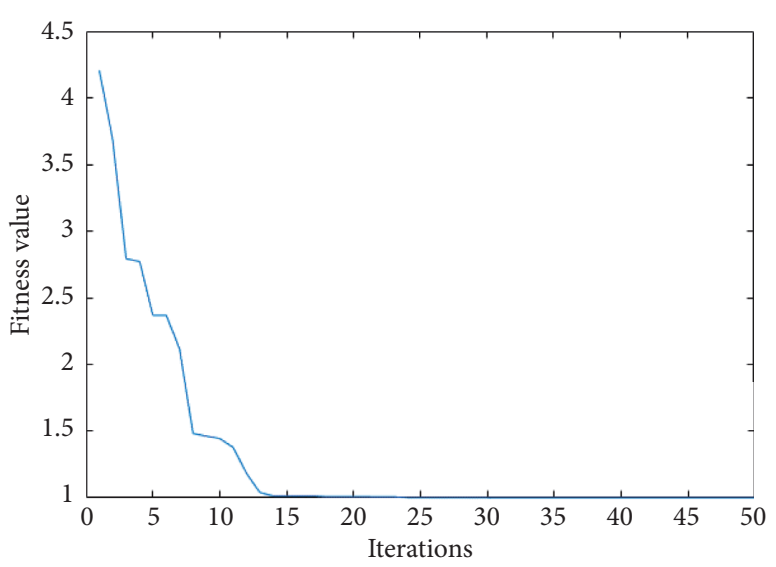

(a)

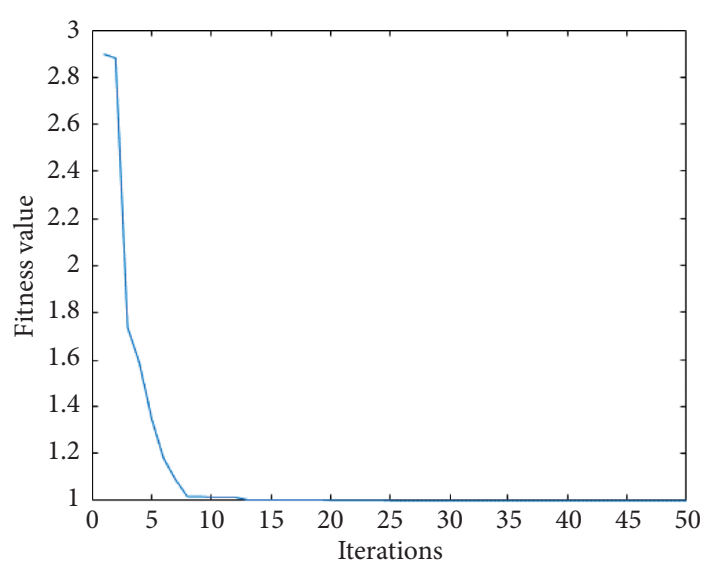

(b)

FIGURE 3: Fitness curve of the optimal particle (a) in the pitch direction and (b) in the yaw direction.

TABLE 1: Optimized results.

\begin{tabular}{lcccc}
\hline Direction & \multicolumn{2}{c}{ SDT matrix } & CN & $\begin{array}{c}\text { Position (coordinates, } \\
\text { unit: } \mathrm{mm})\end{array}$ \\
\hline Pitch & 12153.71 & -4546.85 & 1.6669 & $(0,111, \pm 10)$ \\
& -1037.68 & 10677.85 & & $(0,116, \pm 10)$ \\
Yaw & 8520.84 & -838.03 & 1.0982 & $( \pm 10,121,0)$ \\
& 543.83 & 9304.07 & & $( \pm 10,131,0)$ \\
\hline
\end{tabular}

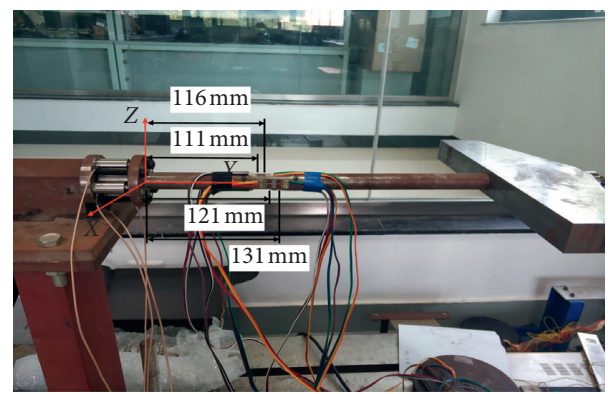

FIgURe 4: Positions of strain gauges.

CZT can be used to calculate the $Z$ transform of a given path. For the $N$ points input sequence $x(n)$, if the transform result is only taken at $N$ equally spaced points on the unit circle, the $Z$ transform at this time is DFT. If the given path is an arc on the unit circle, by increasing the number of output points to make it more than the number of input points, the specified frequency band can be refined.

The $Z$ transformation of a given signal $x(n)$ is defined as

$$
X(z)=\sum_{n=0}^{\infty} x(n) z^{-n}
$$

where $z=e^{s T_{s}}=e^{(\sigma+j \Omega) T_{s}}=e^{\sigma T_{s}} \cdot e^{j \Omega T_{s}}=A e^{j \omega}, s$ is the Laplace variable, $A=e^{\sigma T_{s}}$ is a real number, and $\omega=\Omega T_{s}$ is an angle.

Now modify the $z$ in equation (15), set $z_{r}=\mathrm{AW}^{-r}$, $A=A_{0} e^{j \theta_{0}}$, and $W=W_{0} e^{-j \varphi_{0}}$, then

$$
z_{r}=A_{0} e^{j \theta_{0}} W_{0}^{-r} e^{j \varphi_{0} r}
$$

where $A_{0}$ and $W_{0}$ are any positive real number. Hence, once $A_{0}, W_{0}, \theta_{0}$, and $\varphi_{0}$ is given and $r=0,1, \ldots, \infty$, the points $z_{0}, z_{1}, \ldots, z_{\infty}$ in the $z$-plane can be obtained. Z-transforming these points, we get the definition of CZT:

$$
X\left(z_{r}\right)=\sum_{n=0}^{\infty} x(n) z_{r}^{-n}=\sum_{n=0}^{\infty} x(n) A^{-n} W^{n r} .
$$

Due to $\mathrm{nr}=\left(\left[r^{2}+n^{2}-(r-n)^{2}\right] / 2\right)$, equation (17) can be written as

$$
X\left(z_{r}\right)=\sum_{n=0}^{\infty} x(n) A^{-n} W^{\left(r^{2} / 2\right)} W^{\left(n^{2} / 2\right)} W^{\left(-(r-n)^{2} / 2\right)} .
$$

In fact, equation (18) can be divided into three steps, which are illustrated in Figure 6:

(1) Forming a new sequence $g(n)$ according to the equation

$$
g(n)=x(n) A^{-n} W^{\left(n^{2} / 2\right)}
$$

(2) Convolving $g(n)$ with $W^{\left(-k^{2} / 2\right)}$ to give a sequence

$$
y(n)=\sum_{n=0}^{\infty} g(n) W^{\left(-(r-n)^{2} / 2\right)}
$$

(3) Multiplying $y(n)$ by $W^{\left(r^{2} / 2\right)}$ to give $X\left(z_{r}\right)$

$$
X\left(z_{r}\right)=y(n) W^{\left(r^{2} / 2\right)} .
$$

As shown in Figure 7, when $r=0$ and $z_{0}=A_{0} e^{j \theta_{0}}$, the amplitude of this point in the $z$-plane is $A_{0}$ and the angle is $\theta_{0}$. When $\mathrm{r}=1, z_{1}=A_{0} W_{0}^{-1} e^{j\left(\theta_{0}+\varphi_{0}\right)}$, the amplitude becomes $A_{0} W_{0}^{-1}$, and the angle becomes $\theta_{0}+\varphi_{0}$. It is not difficult to 


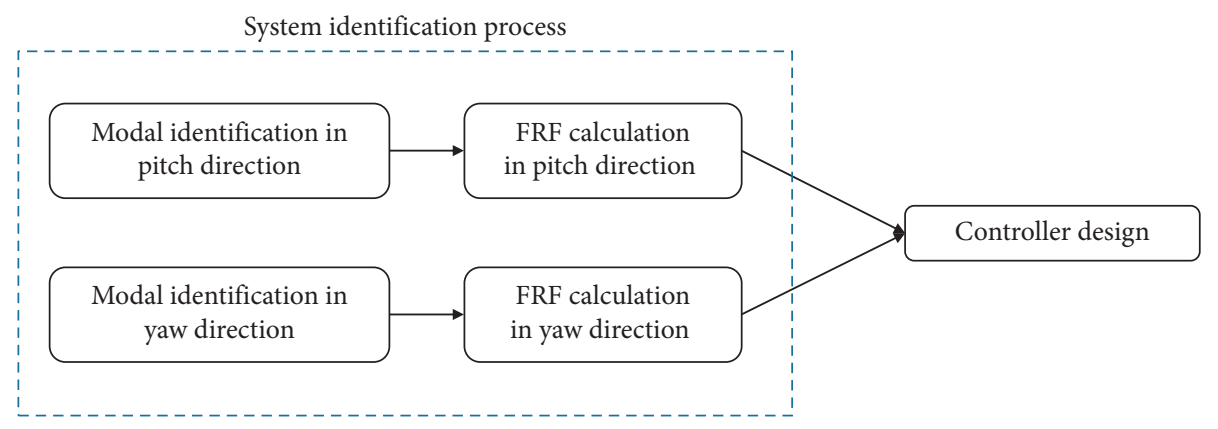

FIgURE 5: System identification process.

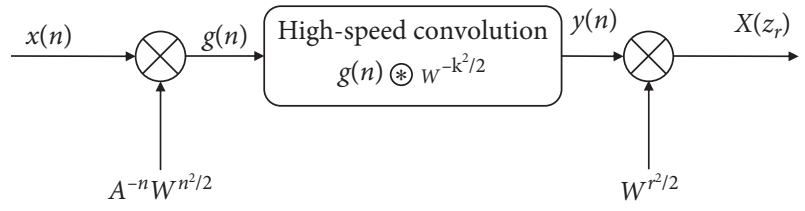

FIGURE 6: Illustration of the steps involved in computing values using the CZT.

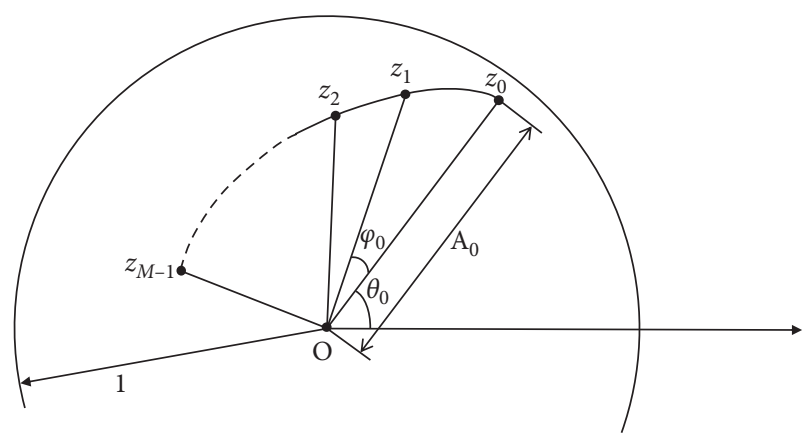

FIgURE 7: Transformation path of CZT.

imagine that when the input $x(n)$ is a sequence of $N$ points and the output $X\left(z_{r}\right)$ is a sequence of $M$ points, with the change in $r$, the points $z_{0}, z_{1}, \ldots, z_{M-1}$ constitute the CZT conversion path, which is a spiral. Meanwhile, the conclusions are summarized as follows:

(1) If $A_{0}>1$, the spiral is outside the unit circle. Otherwise, it is inside the unit circle.

(2) If $W_{0}>1$, then $A_{0} W_{0}^{-1}<A_{0}$; the spiral is internal rotation. Otherwise, it is external rotation.

(3) When $A_{0}=W_{0}=1$, the conversion path of CZT is an arc on the unit circle, and the number of points between the start point and the end point may not be equal to the number of points in the input sequence.

(4) When $A_{0}=W_{0}=1, \theta_{0}=0$, and $M=N$, CZT becomes DFT.

For the $N$-point input sequence $x(n)$ whose sampling frequency is $f_{s}$, in order to obtain the spectrum of $x(n)$, CZT should be implemented on the unit circle, so $A_{0}$ and $W_{0}$ should be taken as $1.0-\left(f_{s} / 2\right)$ on the frequency axis corresponds to the amplitude angle $0-\pi \mathrm{rad}$ on the unit circle. Assuming the frequency band to be refined is
$0 \leq f_{1}<f_{2} \leq\left(f_{s} / 2\right)$ and there are $M$ independent spectral lines, then the corresponding angle on the unit circle is $\left(2 \pi f_{1} / f_{s}\right)-\left(2 \pi f_{2} / f_{s}\right)$, and there are also $M$ points on the arc. It is easy to obtain the calculation conditions for spectrum refinement while using CZT:

$$
\begin{array}{r}
A_{0}=W_{0}=1, \\
\theta_{0}=\frac{2 \pi f_{1}}{f_{s}}, \\
\varphi_{0}=\frac{2 \pi\left(f_{2}-f_{1}\right)}{\left[(M-1) f_{s}\right]} .
\end{array}
$$

When directly calculating the spectrum of $x(n)$ by DFT, there will be $(N / 2)$ independent spectral lines in the frequency range of 0 to $\left(f_{s} / 2\right)$, and the frequency resolution is $\Delta f=\left(f_{s} / N\right)$. After being refined by CZT, there will be $M$ independent spectral lines in the frequency range of $f_{1}$ to $f_{2}$, and the frequency resolution is $\Delta f=\left(\left(f_{2}-f_{1}\right) /(M-1)\right)$. It can be seen that the narrower the fine band and the more the CZT output points, the higher the fine multiple.

\section{Experiments}

To evaluate the effectiveness of SDT matrix as well as system identification and vibration control method, the multidimensional system identification and active vibration control system was built, as shown in Figure 8. All software programs, including excitation and control algorithms, were integrated in the controller (PXIe-8135 with a PXI-7841R field programmable gate array (FPGA) mode card inserted). PXI-7841 R mode card ensures a rapid response, high sampling rate, and low delay of the measuring. Four strain gauge input modules (ADAM-3016) were also included: two of them measure the strain in the pitch direction of the sting, while the other two measure the strain in the yaw direction. In order to amplify the voltage outputted by the controller to the working voltage of piezoelectric stacks, power amplifiers (XE-517.i3) were needed here. The displacement of center of gravity $d_{g}$ and tip of the sting $d_{t}$ were measured by laser displacement sensors (CD5-85), which will be mentioned in the first experiment. The impact hammer (PCB-086C03) is used to ensure that the impact force of contrast experiment is the same every time. 


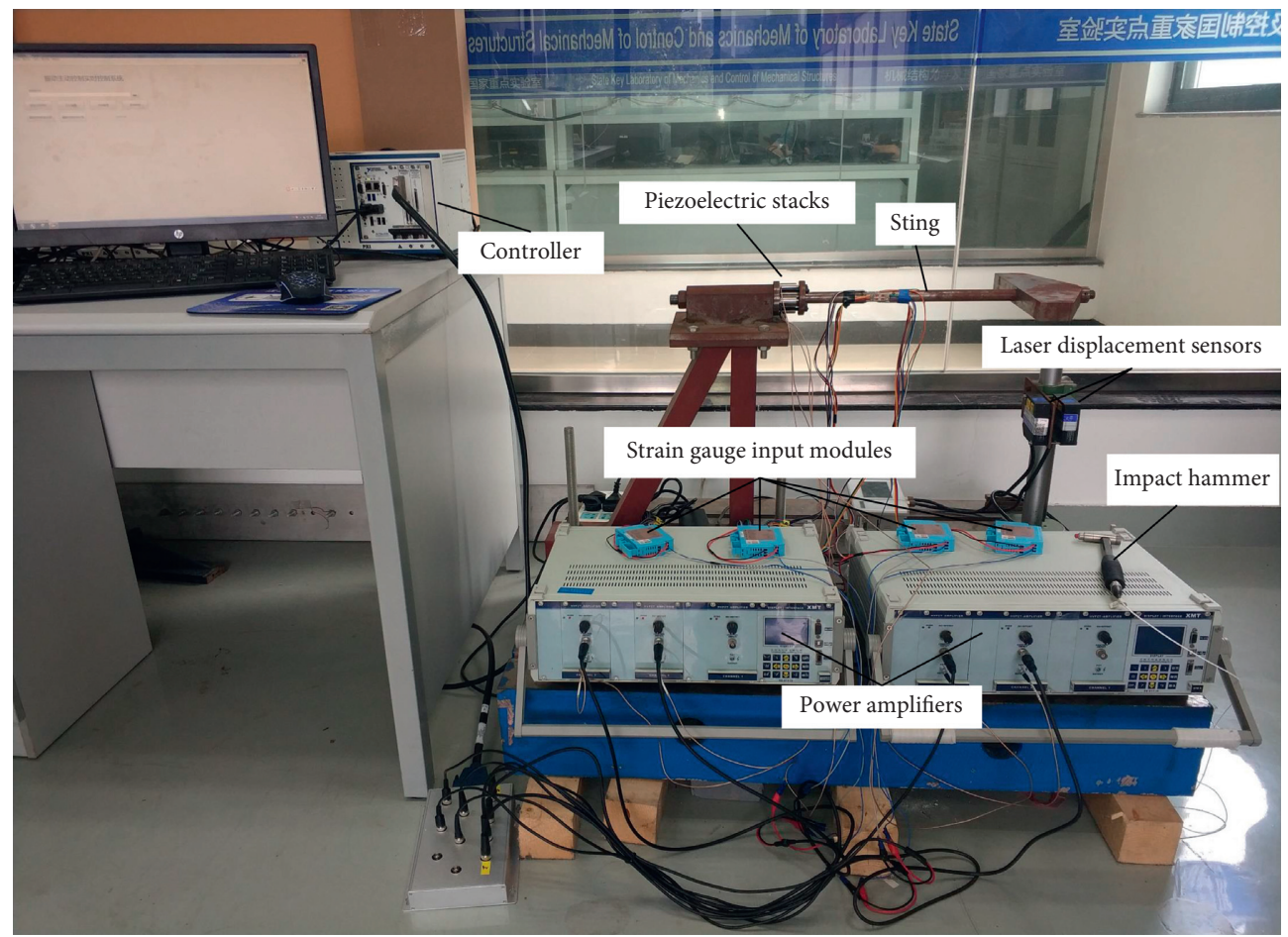

FIGURE 8: Representations of experimental setup.

4.1. Vibration Monitoring Experiment and Results. In this part, piezoelectric stacks were used as the shaker, and schematic diagram is shown in Figure 9. Because the piezoelectric stack can only output thrust and the working voltage cannot be negative, an offset voltage is set before the control to ensure a wider scope of work. Firstly, the piezoelectric stacks excited the vibration under the signal shown in Figure 10 outputted by the controller. The displacement and strain were measured by laser displacement sensors and strain gauges, respectively. Then, two sets of data were collected into the controller at the same time. Finally, the estimated displacement can be calculated by the SDT matrix and compared with the measured displacement.

As shown in Figure 11, the estimated displacement curve basically coincides with the measured displacement curve. The root mean square error (RMSE) is proposed here, which can be written as

$$
\text { RMSE }=\sqrt{\frac{\sum_{n=1}^{N}\left(d_{\text {estimated }}-d_{\text {measured }}\right)^{2}}{N} .}
$$

And RMSE of the results can be seen in Table 2, which is very small. The reason why there is some error at the peak is probably the different sampling rate. The sampling rate of strain gauge input modules is lower than that of laser displacement sensors, which have a certain impact on the results. Overall, the results demonstrate the high accuracy of using SDT matrix to monitor vibration displacement, which lay the foundation for the system identification and vibration control experiments. It is worth mentioning that Figure 12 shows the displacement of center of gravity and tip of the sting is proportional, which is in line with expectations. Therefore, in the subsequent vibration control, only the displacement of tip of the sting is considered.

\subsection{Multidimensional System Identification Experiment and} Results. After confirming the accuracy of the vibration monitor, modal identification of the system was carried out. In practical situations, the vibration energy in each direction is mostly concentrated at the first-order frequency. Furthermore, vibrations above $100 \mathrm{~Hz}$ rarely occur on this structure, so only the first-order mode is considered in pitch and yaw directions, respectively. First of all, the frequency of the excitation generated by the piezoelectric stacks was set from 5 to $50 \mathrm{~Hz}$. The mode frequency is $14.7 \mathrm{~Hz}$ in the yaw direction and $15.8 \mathrm{~Hz}$ in the pitch direction. These have some errors with the finite element simulation, which is mainly caused by the tightness of bolts or the assembling errors. After that, narrow-band excitation around the modal frequency was implemented for more accurate signals. At the same time, the response signals from the strain gauges were collected by the PXI-7841R board card and converted to displacement by the SDT matrix. Finally, the frequency response map based on the CZT could be obtained, from which the amplitude and phase values could also be calculated at the precise mode frequencies. Table 3 gives the system identification results in both two directions.

To verify the effectiveness of the CZT and suppress harmful vibrations of the structure, a controller based on classical PD algorithm is designed here. PID (proportion, integral, and derivative) controller, which is still widely used until now, has many advantages such as simple algorithm, good robustness, and high reliability. The design of PID 


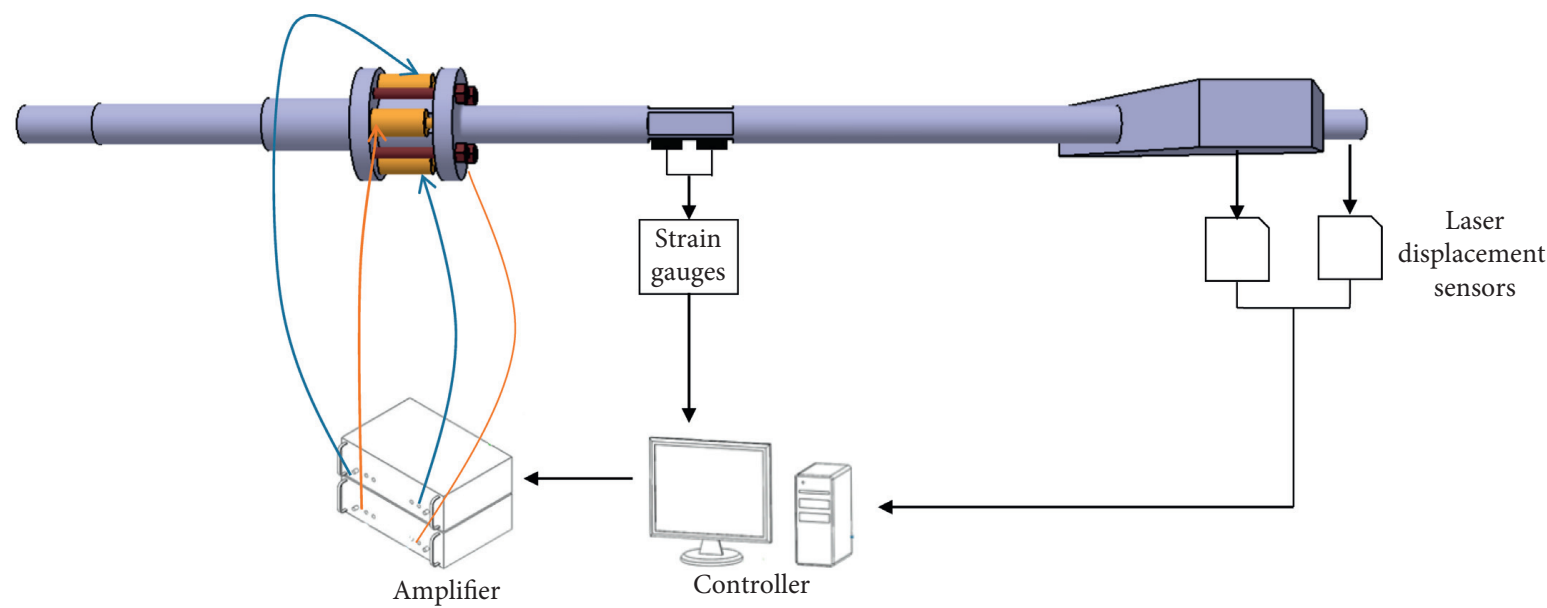

FIGURE 9: Schematic diagram of vibration monitoring experiment.

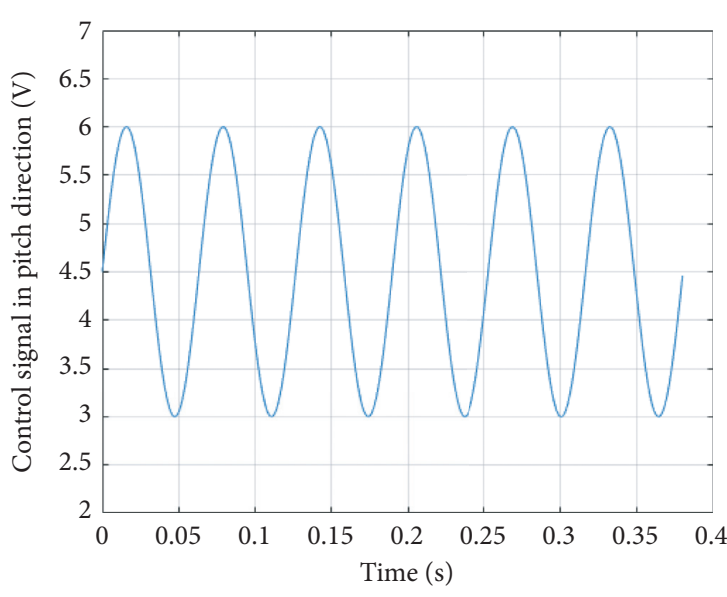

(a)

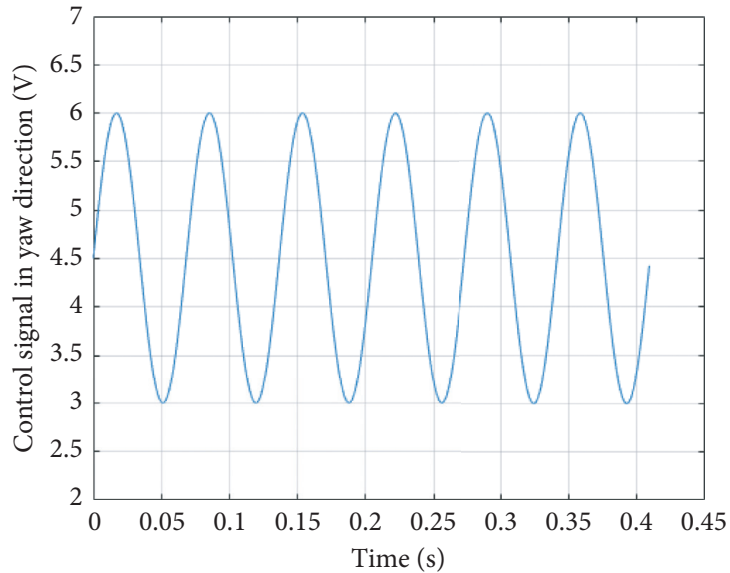

(b)

Figure 10: Excited signal produced by the controller (a) in the pitch direction and (b) in the yaw direction.

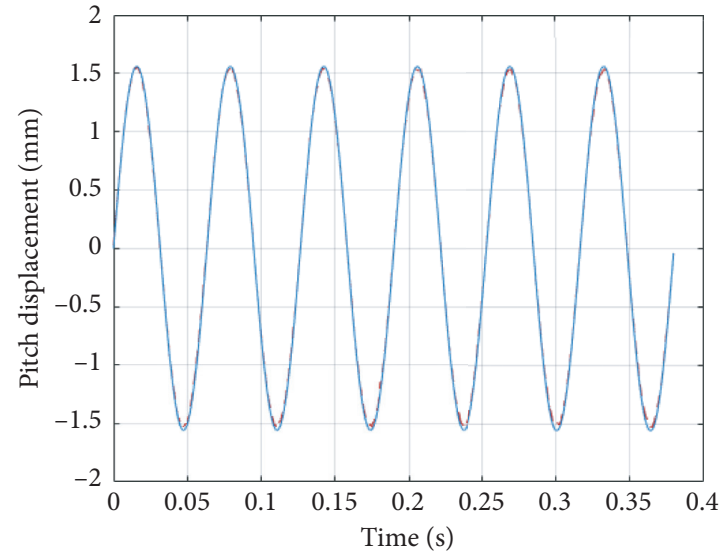

- - - Estimated displacement

— Measured displacement

(a)

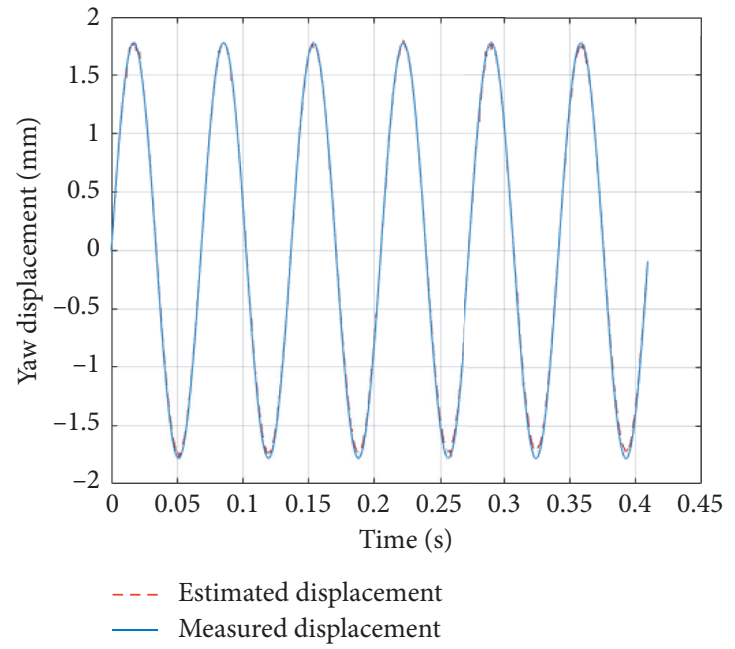

(b) 


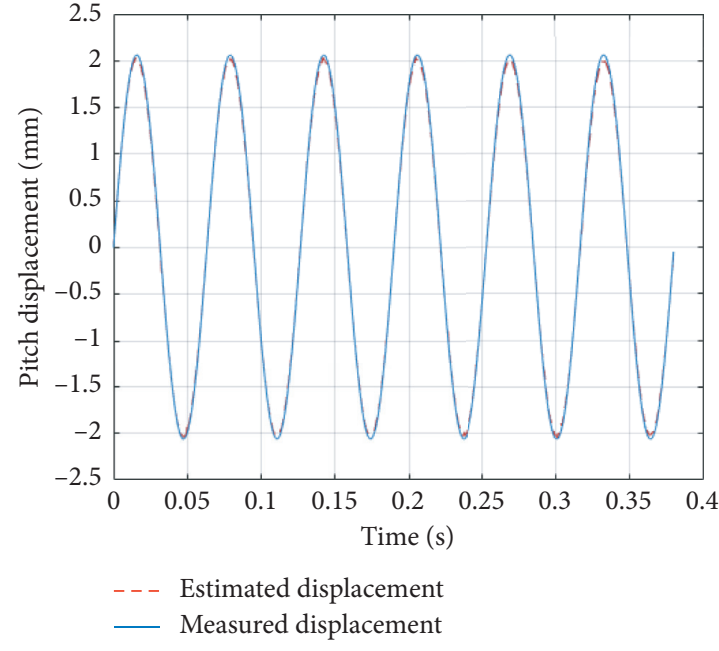

(c)

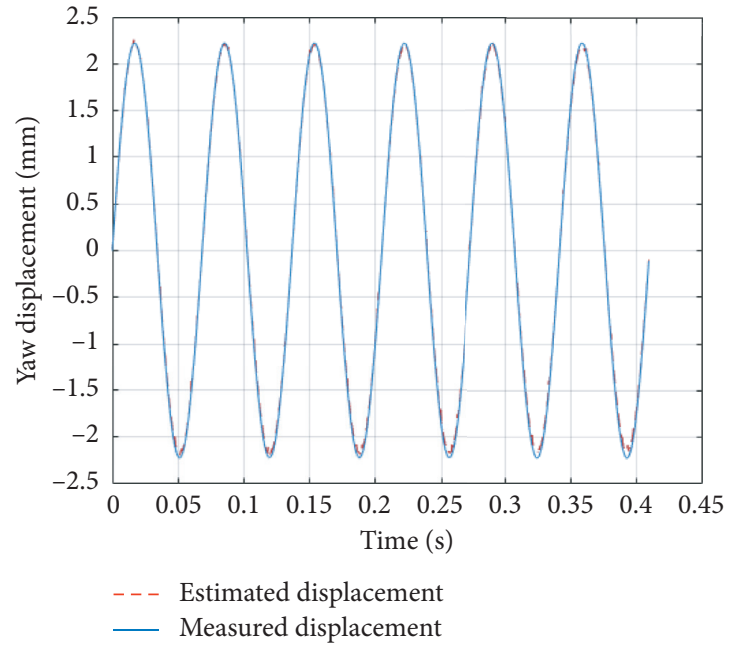

(d)

Figure 11: Vibration monitoring results. Displacement comparison at center of gravity (a) in the pitch direction and (b) in the yaw direction. Displacement comparison at tip of the sting (c) in the pitch direction and (d) in the yaw direction.

TABLE 2: RMSE of vibration monitoring results.

\begin{tabular}{lcc}
\hline Points & Direction & RMSE \\
\hline \multirow{2}{*}{ Center of gravity } & Pitch & 0.0257 \\
& Yaw & 0.0329 \\
Tip of the sting & Pitch & 0.0350 \\
& Yaw & 0.0391 \\
\hline
\end{tabular}

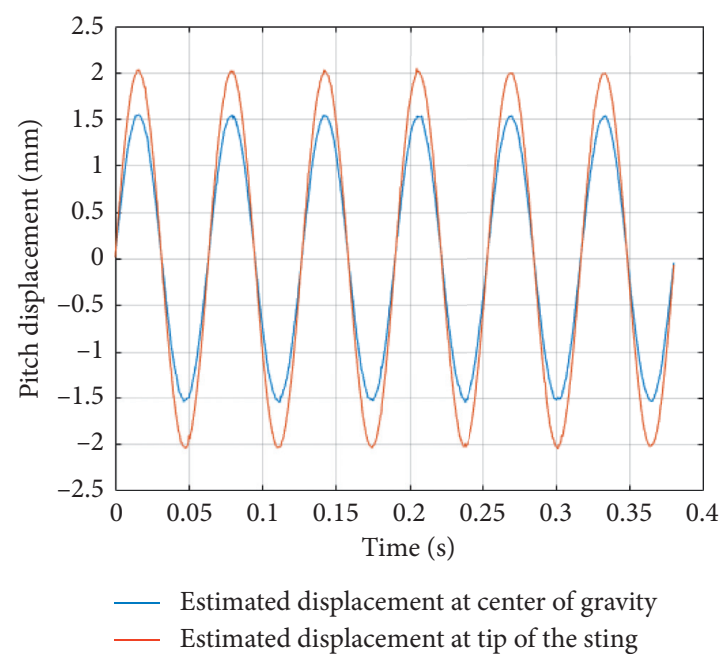

(a)

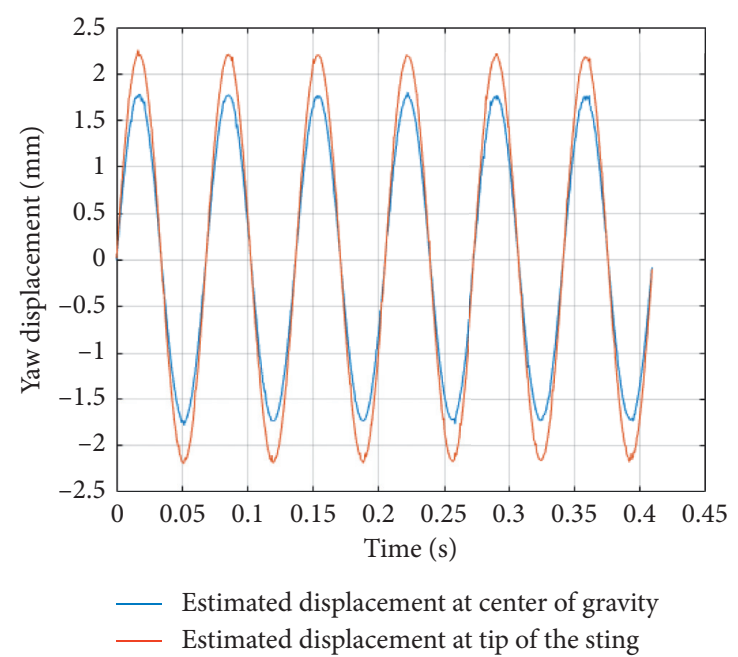

(b)

FIGURE 12: Displacement at center of gravity and tip of the sting (a) in the pitch direction and (b) in the yaw direction.

controller refers more to the setting of control parameters $K_{P}, K_{I}$, and $K_{D}$. The function of $K_{P}$ is to adjust the error signal of the system proportionally. The function of $K_{I}$ is to eliminate the steady-state error of the system and improve the control accuracy. $K_{D}$ is used to overcome the lag, reduce the overshoot, and increase the system stability. Different systems have different values of control parameters due to their inherent characteristics. Only if all parameters take reasonable values can the optimal control performance be guaranteed.

The previous research of our group [22] shows that the sting has the characteristic that, after the vibration attenuation, it will return to the original equilibrium position. Therefore, when designing the controller, there is no need to 
TABLE 3: System identification results.

\begin{tabular}{lccc}
\hline & Frequency $(\mathrm{Hz})$ & Amplitude & Phase $($ deg $)$ \\
\hline Pitch & 15.76 & 3.2601 & 60.007 \\
Yaw & 14.65 & 2.6092 & -75.164 \\
\hline
\end{tabular}

TABle 4: PD parameters.

\begin{tabular}{lcccc}
\hline \multirow{2}{*}{ Value of $g<$} & \multicolumn{2}{c}{ Control parameters for pitch } & \multicolumn{2}{c}{ Control parameters for yaw } \\
& $P$ & $D$ & $P$ & 0.569 \\
0.20 & 0.613 & -0.107 & 0.807 & 0.178 \\
0.15 & 0.869 & -0.152 & 1.282 & 0.252 \\
0.10 & 1.380 & -0.241 & 2.706 & 0.849 \\
0.05 & 2.913 & -0.510 & \\
\hline
\end{tabular}

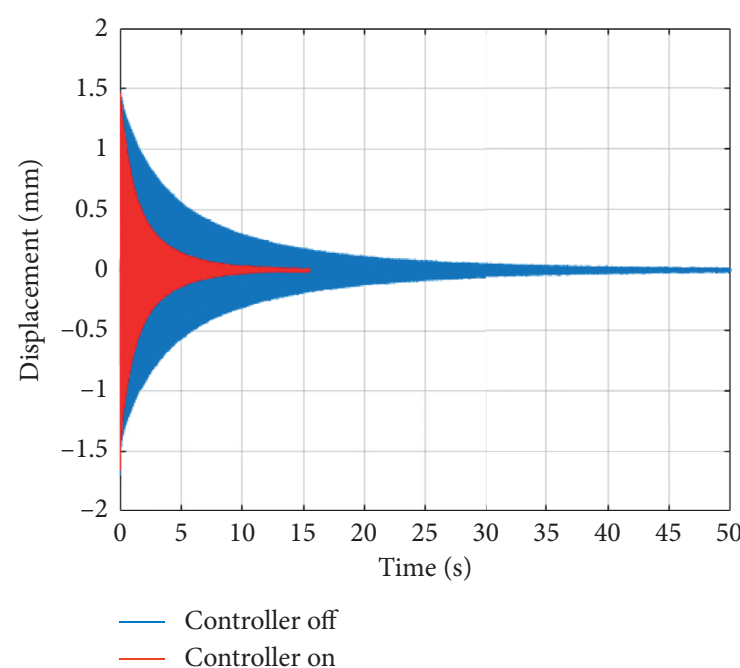

(a)

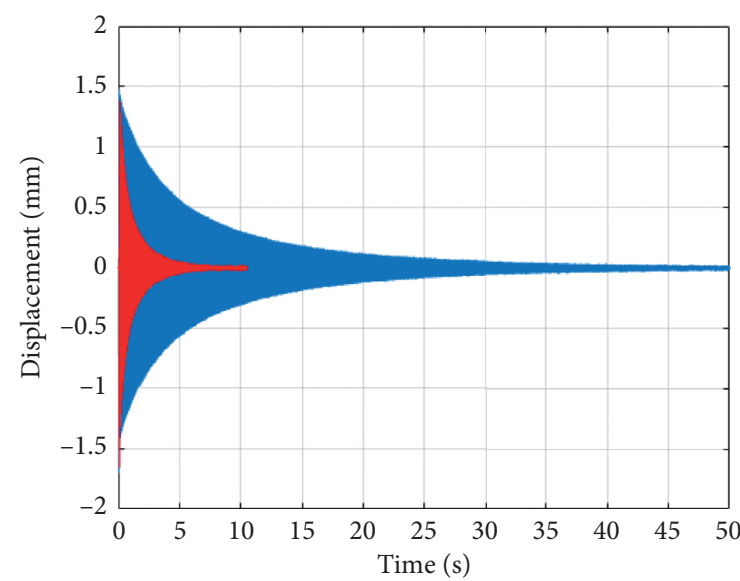

_ Controller off

_ Controller on

(c)

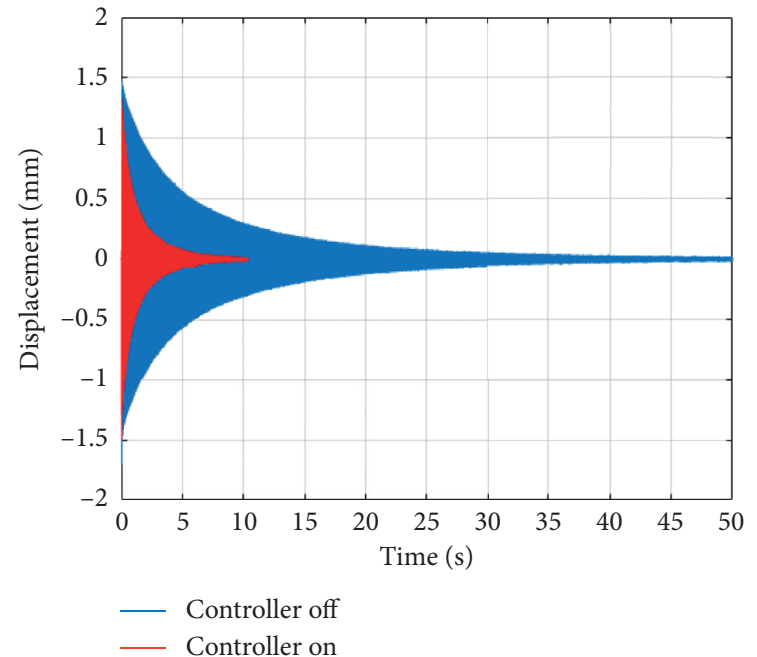

(b)

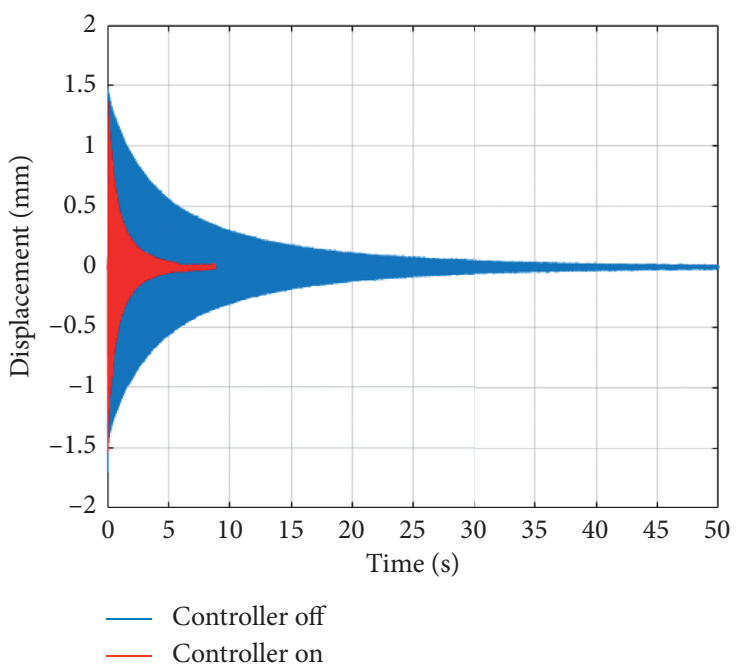

(d)

FIGURE 13: Performance of the controller in the pitch direction under different values: (a) $g=0.20$; (b) $g=0.15$; (c) $g=0.10$; (d) $g=0.05$.

consider I, which is applied to solve the problem of steadystate error, so PD control is used. The expression of the PD controller is

$$
u(t)=K_{P}\left[e(t)+T_{D} \frac{\mathrm{d} e(t)}{\mathrm{d} t}\right]
$$




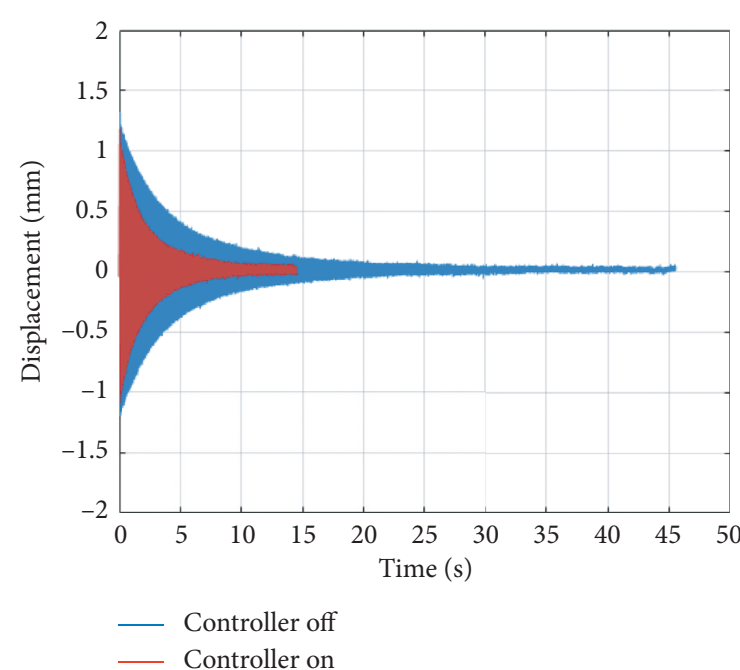

(a)

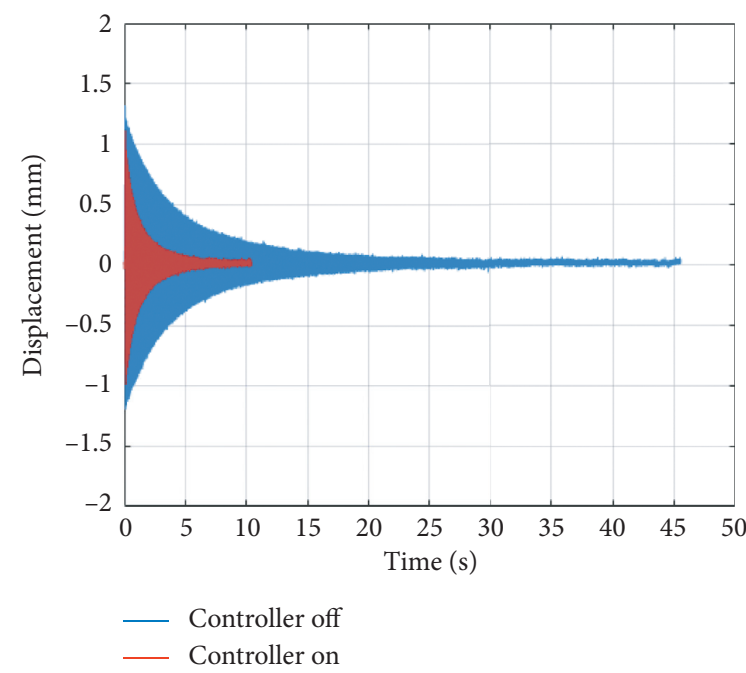

(c)

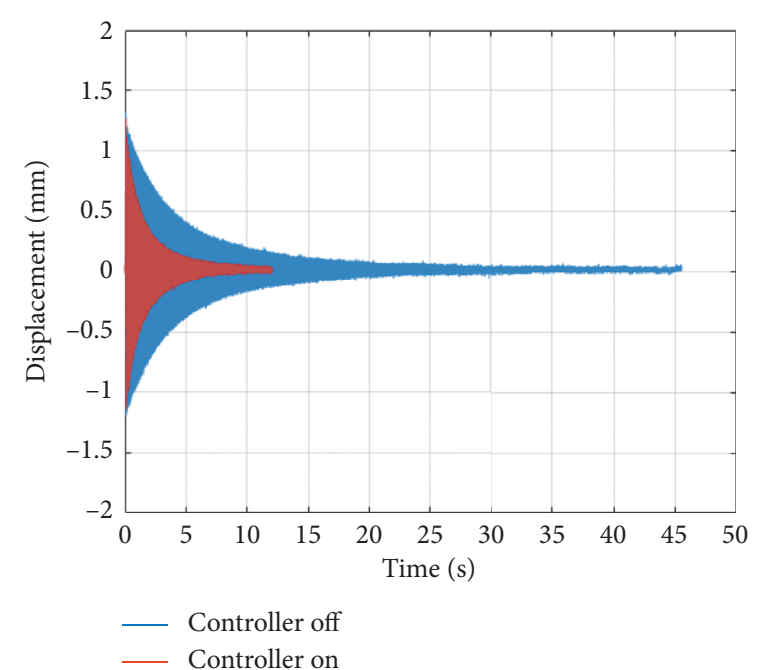

(b)

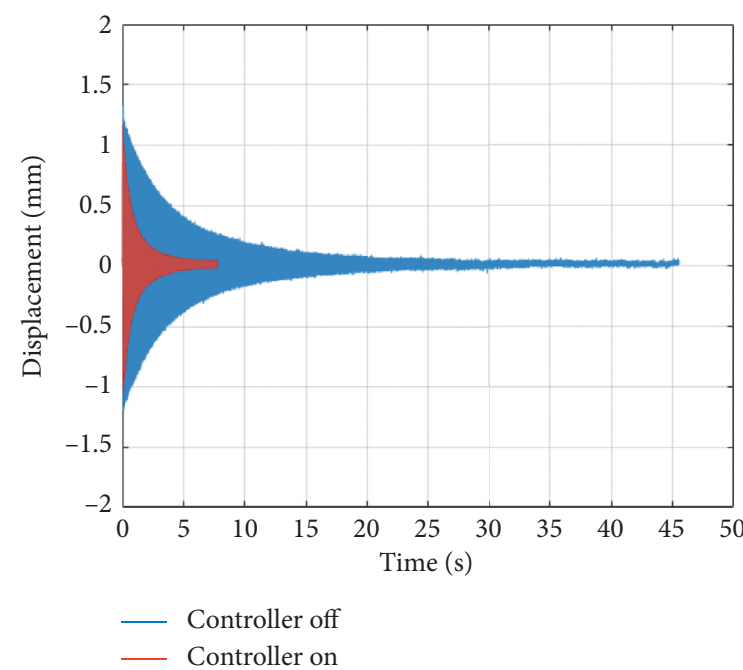

(d)

Figure 14: Performance of the controller in the yaw direction under different values: (a) $g=0.20$; (b) $g=0.15$; (c) $g=0.10$; (d) $g=0.05$.

Then, its transfer function expression is

$$
G_{\mathrm{PD}}(s)=K_{P}\left(1+T_{D} s\right)=K_{P}+K_{D} s
$$

Let $s=j \omega$, then equation (25) can be transformed to

$$
G_{\mathrm{PD}}(j \omega)=K_{P}+j K_{D} \omega .
$$

Since the characteristic signal controlled by this active control system is a sinusoidal signal, it is necessary to correct the phase, especially the phase at the modal frequency. A common phase correction method is

$$
\angle G_{C}\left(j \omega_{r}\right)=-\angle G_{\mathrm{PD}}\left(j \omega_{r}\right),
$$

where $\omega_{r}$ is the mode frequency. Therefore, the proportional and differential parameters of the PD controller satisfy the following equations:

$$
\begin{aligned}
\sqrt{K_{P}^{2}+\left(\omega_{r} K_{D}\right)^{2}} & =\frac{(1 / g)-1}{\left|G_{C}\left(j \omega_{r}\right)\right|}, \\
\arctan \frac{\omega_{r} K_{D}}{K_{P}} & =-\angle G_{C}\left(j \omega_{r}\right) .
\end{aligned}
$$

Among them, $g$ is the control target, which means the ratio of the amplitude after vibration control to the amplitude before control, and the range is $0<g \leq 1$. The smaller the $g$ value, the better the expected control effect. Different PD parameters can be obtained by changing the value of $g$. However, when the set value of $g$ reaches a certain limit, even if the control parameters are correct, the system will diverge. In order to ensure the safety of the experiment, the value of $g$ should not be too small. Therefore, in the experiments, the setting of the $g$ value is always greater than 0.05 . 
TABLe 5: Different damping ratios.

\begin{tabular}{lcc}
\hline Control state & Damping ratio in pitch direction (\%) & Damping ratio in yaw direction (\%) \\
\hline Without control & 0.11 & 0.15 \\
With control, $g=0.20$ & 0.64 & 0.65 \\
With control, $g=0.15$ & 0.83 & 0.78 \\
With control, $g=0.10$ & 0.92 & 0.91 \\
With control, $g=0.05$ & 1.04 & 1.06 \\
\hline
\end{tabular}
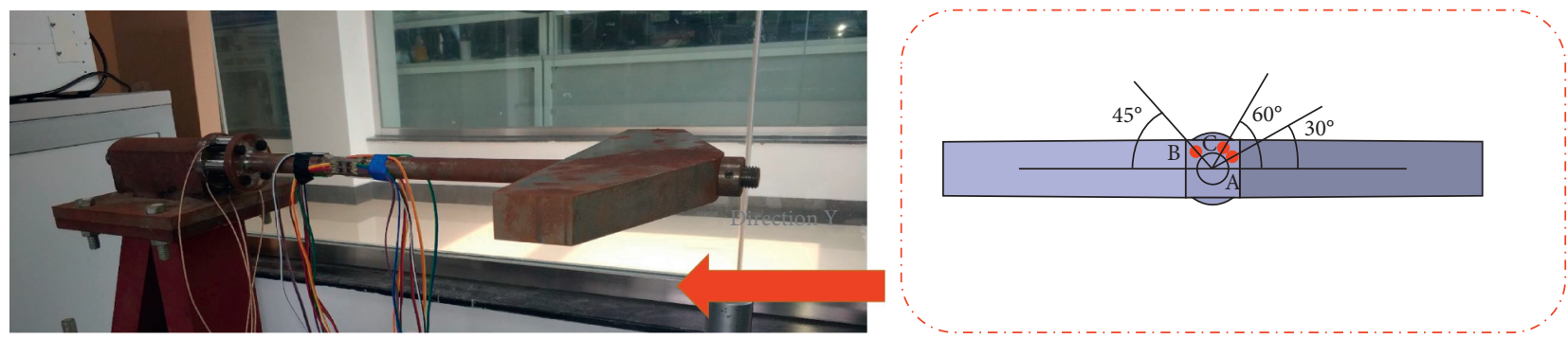

Figure 15: Diagram of the hammering points.

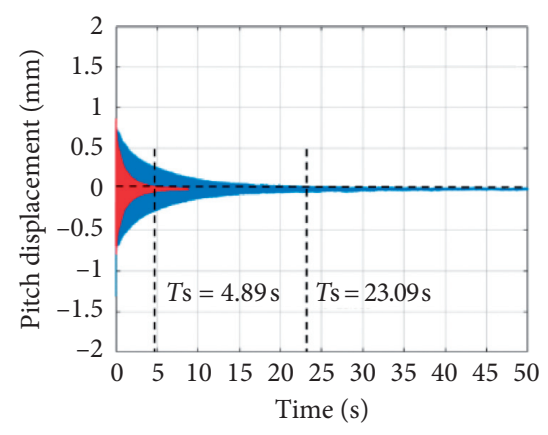

— Controller off

- Controller on

(a)

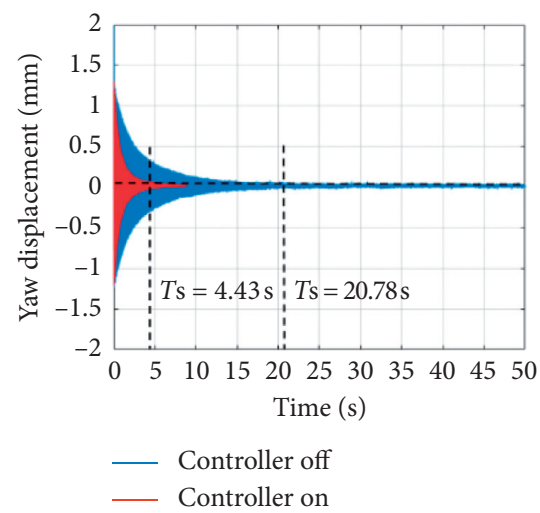

(d)

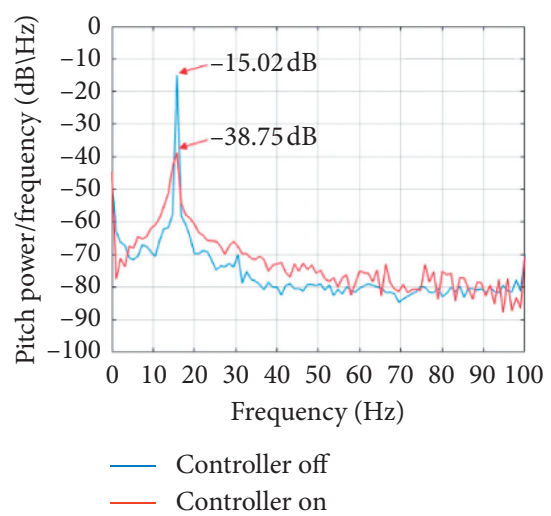

(b)

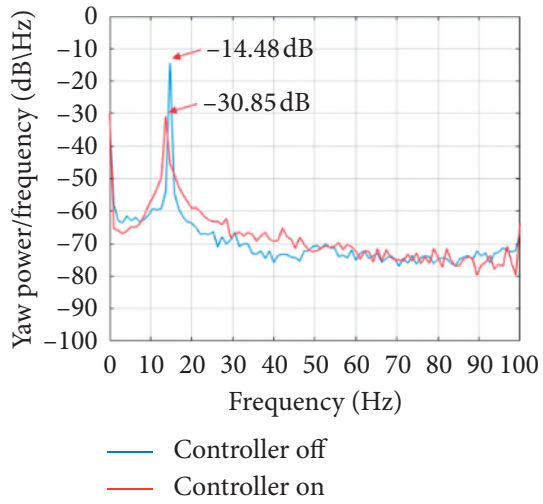

(e)

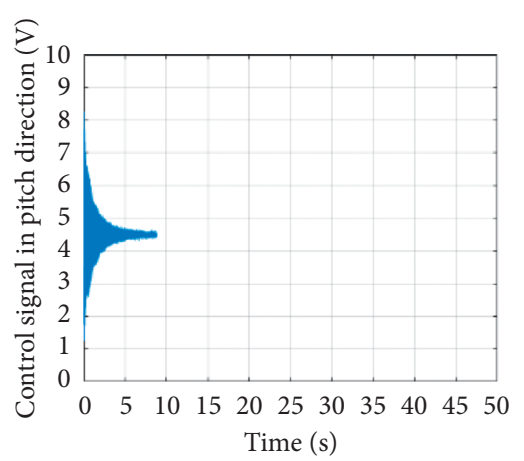

(c)

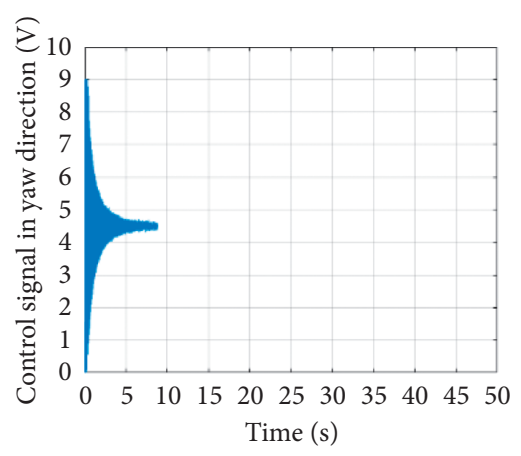

(f)

FIgURE 16: Performance of the controller when the hammering angle is $30^{\circ}$ (A point).

Table 4 gives the PD parameters calculated based on CZT and equation (28). Value of $g$ was set less than 0.2 for both directions in order to get a better control effect. Figure 13 shows the impulse stimulus results in the pitch direction under different values of $g$, and Figure 14 shows the results in the yaw direction.
Obviously, the smaller the value of $g$, the better the control effect. The vibration in pitch and yaw directions lasts for over $40 \mathrm{~s}$ and $25 \mathrm{~s}$ without controller, while the vibration decreased in $4 \mathrm{~s}$ when the controller was on. Table 5 shows different damping ratios calculated by the logarithmic decrement method under different parameters. The 


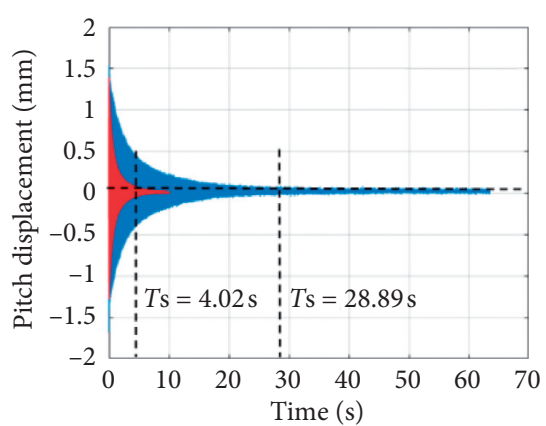

_ Controller off

- Controller on

(a)

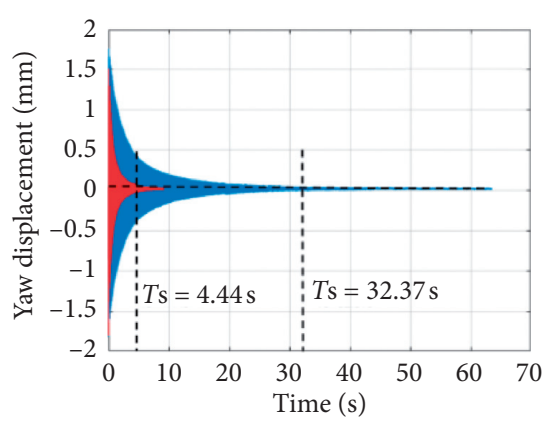

- Controller off

_ Controller on

(d)

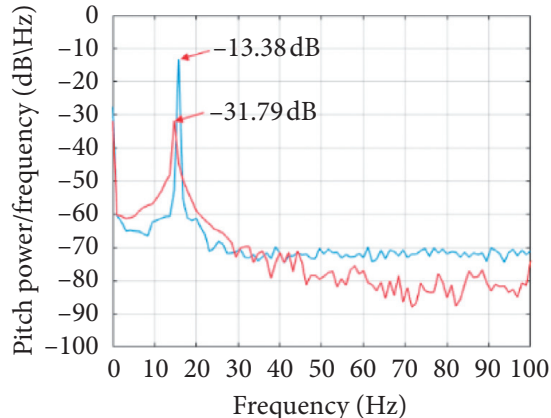

Frequency $(\mathrm{Hz})$

- Controller off

Controller on

(b)

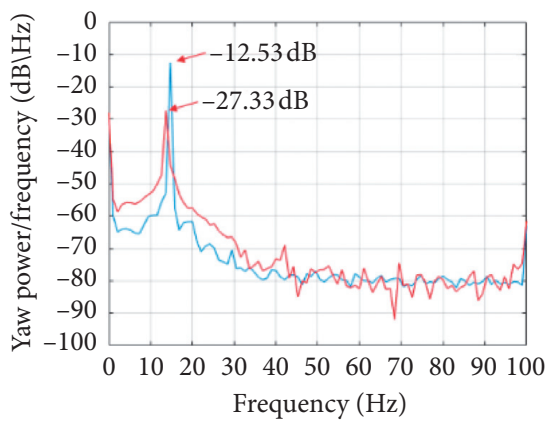

_ Controller off

Controller on

(e)

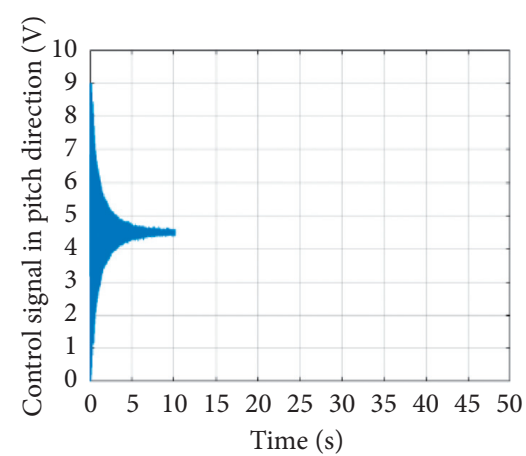

(c)

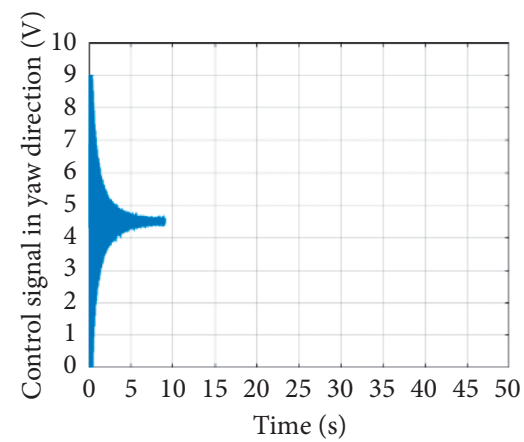

(f)

Figure 17: Performance of the controller when the hammering angle is $45^{\circ}$ (B point).

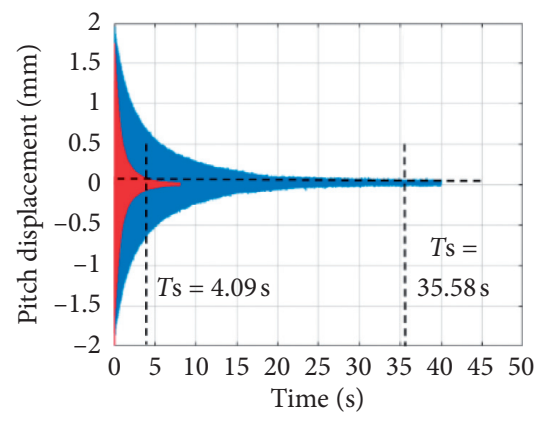

Controller off

Controller on

(a)

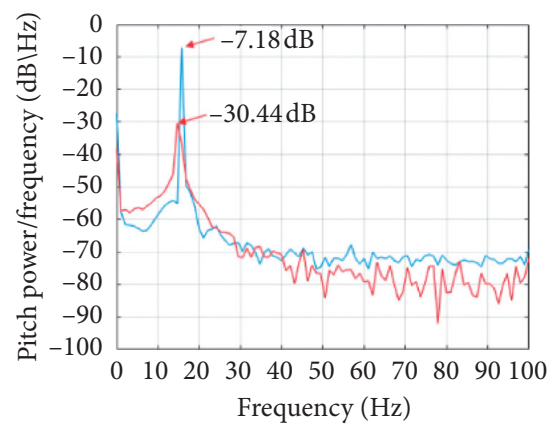

Controller off

Controller on

(b)

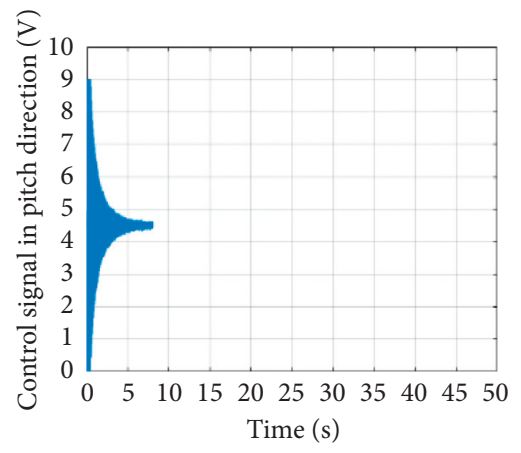

(c)

Figure 18: Continued. 


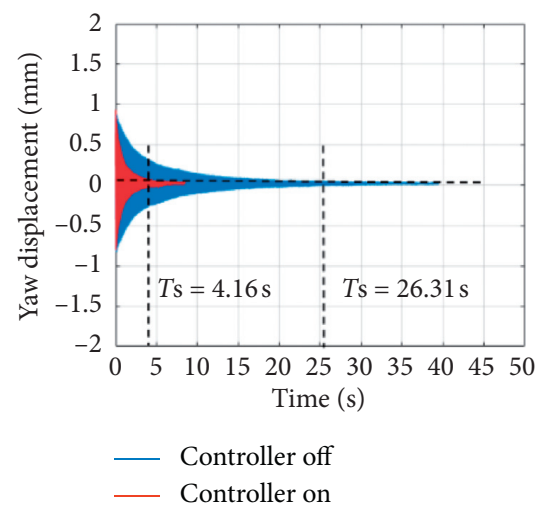

(d)

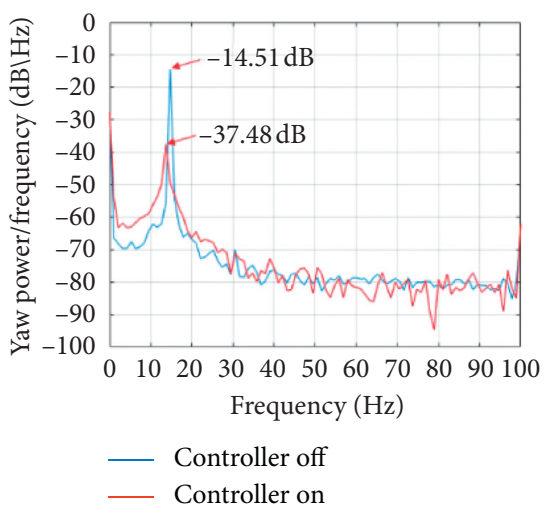

(e)

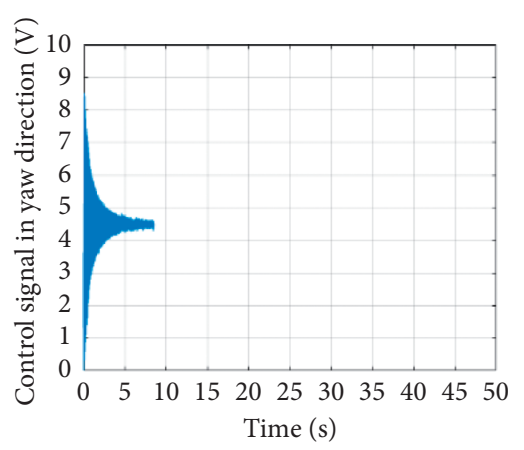

(f)

Figure 18: Performance of the controller when the hammering angle is $60^{\circ}$ (C point).

TABLE 6: Contrast results at different points.

\begin{tabular}{|c|c|c|c|c|c|}
\hline $\begin{array}{l}\text { Hammering } \\
\text { point }\end{array}$ & $\begin{array}{l}\text { Vibration direction } \\
\text { pitch/yaw }\end{array}$ & $\begin{array}{c}\text { Active controller } \\
\text { on/off }\end{array}$ & $\begin{array}{c}\text { Damping ratio } \\
(\%)\end{array}$ & $\begin{array}{l}\text { Attenuation time } \\
\text { (s) }\end{array}$ & $\begin{array}{c}\text { Spectral } \\
\text { attenuation } \\
(\mathrm{dB} / \mathrm{Hz})\end{array}$ \\
\hline \multirow{4}{*}{$30^{\circ}$ (A point) } & \multirow{2}{*}{ Pitch } & Off & 0.16 & 23.09 & \multirow{2}{*}{23.73} \\
\hline & & On & 1.09 & 4.89 & \\
\hline & \multirow{2}{*}{ Yaw } & Off & 0.11 & 20.78 & \multirow{2}{*}{16.37} \\
\hline & & On & 1.27 & 4.43 & \\
\hline \multirow{4}{*}{$45^{\circ}$ (B point) } & \multirow{2}{*}{ Pitch } & Off & 0.10 & 28.89 & \multirow{2}{*}{18.41} \\
\hline & & On & 1.27 & 4.02 & \\
\hline & \multirow{2}{*}{ Yaw } & Off & 0.09 & 32.37 & \multirow{2}{*}{14.80} \\
\hline & & On & 1.42 & 4.44 & \\
\hline \multirow{4}{*}{$60^{\circ}$ (C point $)$} & \multirow{2}{*}{ Pitch } & Off & 0.12 & 35.58 & \multirow{2}{*}{23.26} \\
\hline & & On & 1.84 & 4.09 & \\
\hline & \multirow{2}{*}{ Yaw } & Off & 0.08 & 25.31 & \multirow{2}{*}{22.97} \\
\hline & & On & 1.21 & 4.16 & \\
\hline
\end{tabular}

damping ratio in the pitch direction is strengthened 9.5 times (from $0.11 \%$ to $1.04 \%$ ) and 7.1 times (from $0.15 \%$ to $1.06 \%)$ in the yaw direction.

Summarizing the results, it becomes evident that the controller designed based on the vibration monitoring method and CZT performs very well, and the effect of suppressing vibration is confirmed.

\subsection{Multidimensional Vibration Control Experiment and} Results. Based on the multidimensional vibration controller designed, vibration control experiments were carried out. In this part, the broadband random dynamic wind load was simulated by hammering. Three hammering points $\mathrm{A}, \mathrm{B}$, and $\mathrm{C}$ is shown from the $Y$ direction in Figure 15, and the angle between the knocking direction and horizontal plane is $30^{\circ}$, $45^{\circ}$, and $60^{\circ}$, respectively. The contrast effects in pitch and yaw directions with multidimensional vibration control system off and on are shown in Figures 16-18.

Table 6 shows the contrast results when the system was hammered at $\mathrm{A}, \mathrm{B}$, and $\mathrm{C}$. For any point, the vibration is damped within $4.89 \mathrm{~s}$, the damping ratio is strengthened more than 6.82 times, and the energy is attenuated over $14.8 \mathrm{~dB} / \mathrm{Hz}$. Control effect can also be seen by the control signal applied to the piezoelectric stacks for the control signal is attenuated quickly. The best control effect appears at $\mathrm{C}$ point, where damping ratio is improved more than 15 times, attenuation time is shortened over 8.6 times, and attenuation of spectral is around $23 \mathrm{~dB} / \mathrm{Hz}$.

\section{Conclusions}

Vibration damping has always been a hotspot, but research on multidimensional vibration control is scarce. In this paper, a wind tunnel cantilever sting was processed as well as the vibration monitoring method based on the SDT matrix. With this method, vibration displacement of the model can be estimated easily by the strain signals without complex instruments like laser displacement sensors or accelerometers. Then, multidimensional system identification based on CZT was applied, and parameters of the controller were obtained. While other methods might have deviations, the CZT-based refinement method was adopted and suitable for multidimensional FRF calculations. After a great number of experiments completed in the laboratory, the exactness of the vibration monitoring method and system identification was proved. The damping ratio of the system is increased by more than 6.82 times, and the spectrum power is reduced 
over $14.8 \mathrm{~dB} / \mathrm{Hz}$, which means the vibration in pitch and yaw directions was attenuated apparently. Therefore, the multidimensional system identification and active control system could be a powerful tool to monitor vibration signals, identify frequency response functions, and reduce the errors of collected signals in wind tunnel tests.

\section{Data Availability}

The data used to support the findings of this study are available from the corresponding author upon request.

\section{Conflicts of Interest}

The authors declare that there are no conflicts of interest regarding the publication of this paper.

\section{Acknowledgments}

This study was funded by the National Natural Science Foundation of China (no. 11872207), the Aeronautical Science Foundation of China (no. 20180952007), the Research Fund of State Key Laboratory of Mechanics and Control of Mechanical Structures (no. MCMS-I-0520G01), and the Postgraduate Research and Practice Innovation Program of Jiangsu Province (no. KYCX19_0170).

\section{References}

[1] M. L. Post and T. C. Corke, "Separation control on HIgh angle of attack airfoil using plasma actuators," AIAA Journal, vol. 42, no. 11, pp. 2177-2184, 2004.

[2] Y. Dai, L. Zhang, Z. Zhao et al., "Wind-tunnel evaluation for an active sting damper using multimodal neural networks," AIAA Journal, vol. 58, no. 4, pp. 1-10, 2020.

[3] M. Zhou, W. Liu, L. Tang et al., "Multidimensional vibration suppression method with piezoelectric control for wind tunnel models," Sensors, vol. 19, no. 18, p. 3998, 2019.

[4] W. B. Igoe and F. J. Capone, Reduction of Wind Tunnel Model Vibration By Means of a Tuned Damped Vibration Absorber Installed in a Model, NASA Langley Research Center, Washington, DC, USA, July 1968.

[5] T. Igusa and K. Xu, "Vibration control using multiple tuned mass dampers," Journal of Sound and Vibration, vol. 175, no. 4, pp. 491-503, 1994.

[6] R. Glaese, G. Bales, S. Hsu et al., "Reduction of dynamic response of a wind tunnel sting mount using a hub damper unit," in Proceedings of the Aiaa Aerospace Sciences Meeting Including the New Horizons Forum \& Aerospace Exposition, Orlando, FL, USA, January 2010.

[7] H. Fehren, U. Gnauert, R. Wimmel et al., "Validation testing with the active damping system in the European Transonic Wind tunnel," in Proceedings of the Aerospace Sciences Meeting \& Exhibit, Reno, NV, USA, January 2001.

[8] G. Hefer, "Etw-a facility for high Reynolds number testing," IUTAM Symposium Transsonicum IV, vol. 73, pp. 157-164, 2003.

[9] D. Schimanski and G. Hefer, "Recent aspects of high Reynolds number data quality and capabilities at the European Transonic Wind tunnel," in Proceedings of the Aerospace Sciences Meeting \& Exhibit, Reno, NV, USA, January 2013.

[10] S. Balakrishna, H. Houlden, D. Butler et al., "Development of a wind tunnel active vibration reduction system," in
Proceedings of the 45th AIAA Aerospace Sciences Meeting and Exhibit, Reno, NV, USA, January 2007.

[11] S. Balakrishna, D. H. Butler, R. White, and W. A. Kilgore, "Active damping of sting vibrations in transonic wind tunnel testing," Report No.: AIAA-2008-840, AIAA, Reston, VA, USA, 2008.

[12] M. Acheson and S. Balakrishna, "Effects of active sting damping on common research model data quality," Report No.: AIAA-2011-878, AIAA, Reston, VA, USA, 2011.

[13] M. B. Rivers and S. Balakrishna, "NASA common research model test envelope extension with active sting damping at NTF," Report No.: AIAA-2014-3135, AIAA, Reston, VA, USA, 2014.

[14] M. Jovanovic Miroslav, M. Simonovic Aleksandar, D. Zoric Nemanja et al., "Experimental studies on active vibration control of a smart composite beam using a PID controller," Smart Materials and Structures, vol. 22, no. 11, p. 115038, 2013.

[15] X. Shen, L. Zhang, and Y. Chen, "Development and validation research of an active sting damper in a transonic acoustic wind tunnel," in Proceedings of the 2018 15th International Bhurban Conference on Applied Sciences and Technology (IBCAST), Islamabad, Pakistan, January 2018.

[16] X. Shen, Y. Dai, M. Chen et al., "Active vibration control of the sting used in wind tunnel: comparison of three control algorithms," Shock and Vibration, vol. 2018, no. 9, pp. 1905049.1-1905049.10, 2018.

[17] J. Chen, X. Shen, F. Tu et al., "Experimental research on an active sting damper in a low speed acoustic wind tunnel," Shock and Vibration, vol. 2014, no. 1, pp. 524351.1-524351.10, 2014.

[18] Y. Li, X. Shen, and Y. Chen, "Design, experiment and verification of a refined resonance method for property measurement of piezoelectric stack," Smart Materials and Structures, vol. 28, 2018.

[19] W. Liu, M. D. Zhou, Z. Q. Wen et al., "An active damping vibration control system for wind tunnel models," Chinese Journal of Aeronautics, vol. 32, no. 9, pp. 2109-2120, 2019.

[20] S. Rapp, L. H. Kang, J. H. Han et al., "Displacement field estimation for a two-dimensional structure using fiber Bragg grating sensors," Smart Materials and Structures, vol. 18, no. 2, Article ID 025006, 2009.

[21] Y. H. Shi and R. C. Eberhart, "Empirical study of particle swarm optimization," in Proceedings of the 1999 Congress on Evolutionary Computation-CEC99 (Cat. No. 99TH8406), IEEE, Washington, DC, USA, July 2002.

[22] Y. Dai, X. Shen, L. Zhang, Y. Yu et al., "System identification and experiment evaluation of a piezoelectric-based sting damper in a transonic wind tunnel," Review of Scientific Instruments, vol. 90, no. 7, Article ID 075102, 2019. 